\title{
Uber Ablauf und Altersstellung altquartärer Verschüttungen im Maintal und nächst dem Donautal bei Regensburg
}

\author{
Von KarL Brunnacker, Köln
}

\author{
Mit 1 Abbildung und 1 Tabelle im Text
}

\begin{abstract}
$\mathrm{Zus}$ a m men f a s u $\mathrm{ng}$. Sowohl im Tal des Regen nächst seiner Einmündung in die Donau bei Regensburg wie im unteren Maintal bei Marktheidenfeld sind mächtige altquartäre Talverschüttungen aufgeschlossen. Sie lassen sich jeweils in eine ältere Akkumulationsphase, eine nachfolgende Auephase und in eine abschließende jüngere Akkumulationsphase aufgliedern. Einer im Maintal nachfolgenden Erosionsperiode entspricht bei Regensburg ein extremer Pseudogley. Darüber folgen Gehängeablagerungen und schließlich Löß unterschiedlicher Fazies mit drei zwischengeschalteten interglazialen Parabraunerden.

Damit liegt in beiden Talsystemen die große Verschüttungsperiode vor der viertletzten Eiszeit. Auch die der Jüngeren Steppenzeit zugeordnete Säugetierfauna von Randersacker gehört in die (jüngere?) Verschüttungsphase. Die Ursache der offensichtlich nicht allein auf das untere Mainund Neckartal beschränkten Talverschüttung wird in einer allgemeinen Hebungstendenz der Mittelgebirge vor der Günzeiszeit vermutet.

$\mathrm{Summary}$. Extensive accumulations of old Quaternary rivers are exposed in both the valley of the Regen next to its mouth to the Danube near Regensburg and in the valley of the Main near Marktheidenfeld. They can be divided into an older phase of accumulation, a following interval of stagnation (peat and plain soil formation) and a younger period of sand deposition. This is followed by erosion along the river Main and by soil formation (extreme Pseudogley) in the valley of the river Regen. Both regions are characterized by covering colluvium and löss of different facies. Three interglacial soils („Parabraunerde“) occurring in between the sequence offer means of correlation. The river sediments can be dated before the last four ice-ages. Vertebrate fossils, found in these sediments of the Main near Randersacker, indicate "Jüngere Steppenzeit" (according to K. D. Adam 1953).
\end{abstract}

Accumulation of such extensive river deposits probably was caused by epirogenetic uplift of the Hercynian mountains in southern Germany.

Die Vorstellung einer wenigstens streckenweise wirksamen starken Eintiefung des Maintales mit nachfolgender mächtiger Verschüttung im älteren Quartär wurde bereits durch L. Hirsch (1940) entwickelt. Ausgehend vom Volkacher Gebiet wurde sie jedoch erst durch A. WuRM (1956) bewiesen und schließlich durch E. RuTTE (1958) auch paläontologisch im Bereich von Randersacker bei Würzburg innerhalb der altquartären Säugetierfauna Süddeutschlands (K. D. ADAm 1953) genauer fixiert. Schließlich hat H. Körber (1962) in einer umfassenden Darstellung der Entstehung des Maintales deren Verbreitung und Beziehung zu den älteren wie zu den jüngeren Terrassen festgelegt. Die paläontologische Bearbeitung durch E. Rutre (1958) ergab für die Verschüttung ein Günz/Mindelinterglaziales Alter, das nachfolgend von H. KöRBER (1962) als Ausgangsbasis der Terrasseneinstufung genommen wurde. Die von H. KöRBER (1962, S. 155/156) gegebene stratigraphische Ubersicht deutet jedoch damit insofern Komplikationen an, als die jüngere Terrassenfolge die Zwischenschaltung wenigstens eines „Interstadials“ erfordert, das hinsichtlich seiner geomorphologischen Wirksamkeit den interglazialen Erosionsperioden ungefähr gleichwertig war.

Neuerdings wurde ferner aus dem Regental bei Regensburg eine weitere alte Talverschüttung bekannt gemacht, die gemäß ihrer Deckschichtenfolge und der dazwischenlagernden Paläoböden vor die Günzeiszeit einzustufen ist. Dieser Abschnitt wurde der 


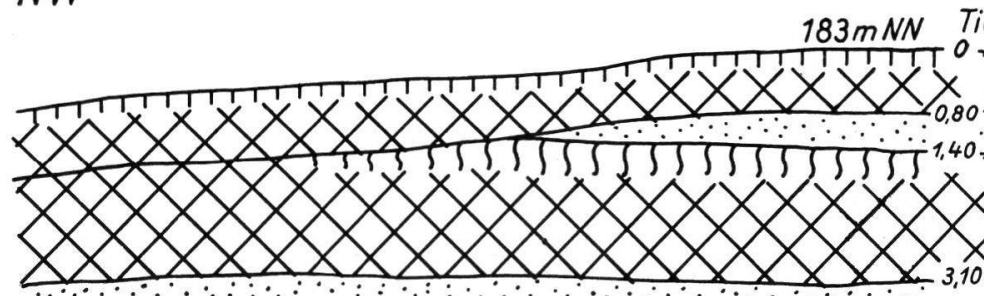

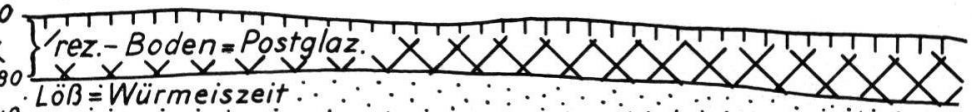

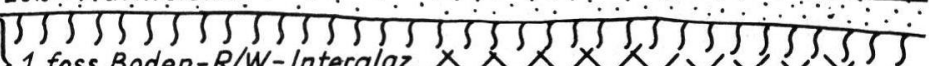

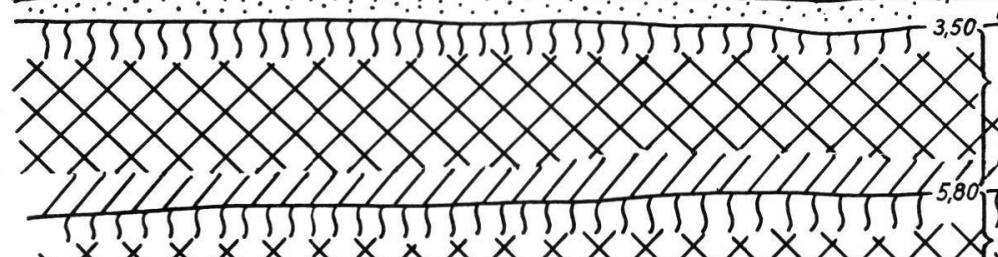

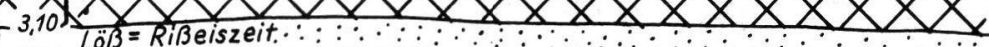

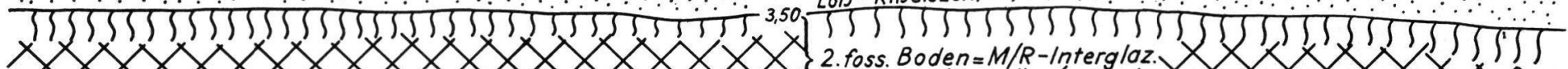

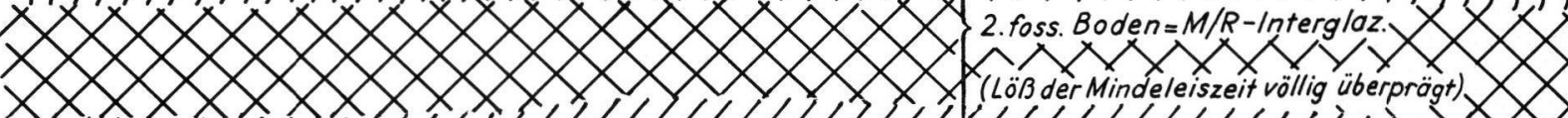

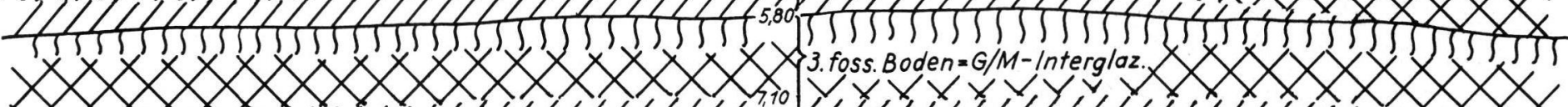
(1)

Ooter Ton als zwischenmittel ?"

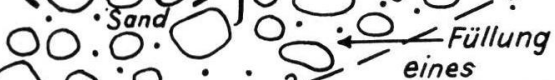

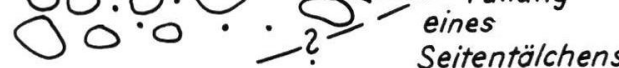

ح $\overbrace{0}^{0} 0$.

Löß? = Günzeiszeit

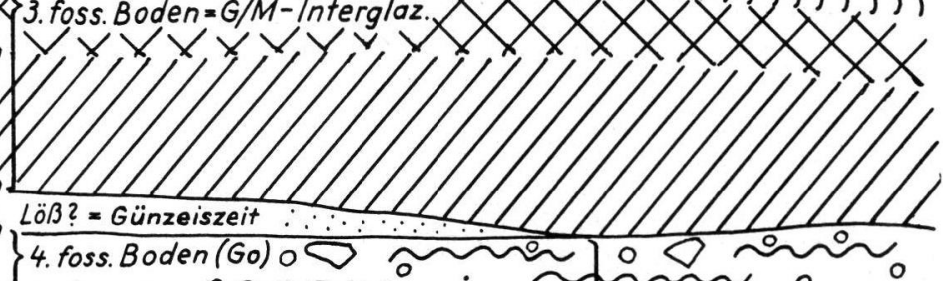

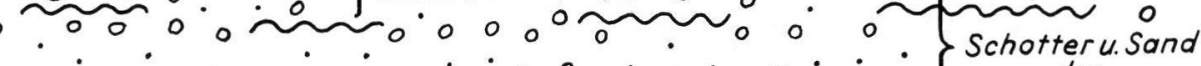

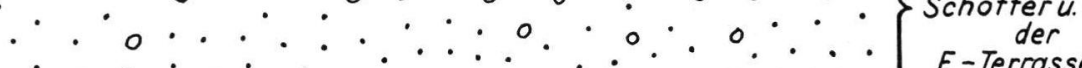

$\cdot \begin{array}{cccc}\cdot & 0 & 0 & 0 \\ 0 & 0 & 0\end{array}$

- $0 \cdot 0.0^{\circ} \cdot 0$ Erosionsdiskordanz 000

$$
.0
$$

Abb. 1. Profil der Ziegeleigrube von Marktheidenfeld.

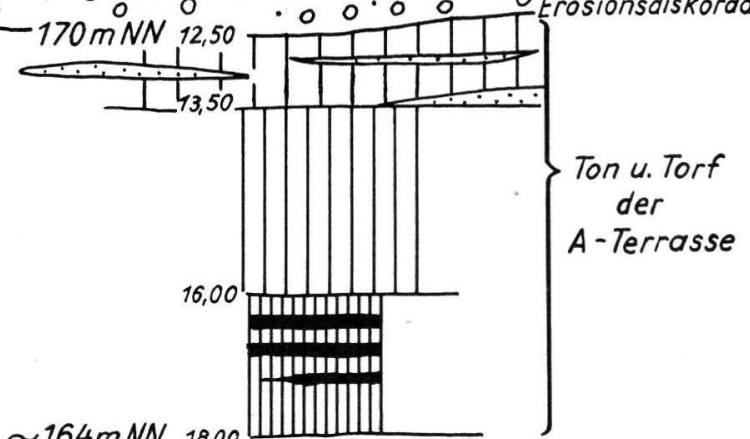


"Warmzeit mit dem Riesenboden bzw. dem Komplex der Riesenböden" zugeordnet (K. Brunnacker 1964, vgl. auch 1962).

Die Regensburger Befunde mit denen des Maintales zu vergleichen, liegt, wie schon angedeutet (K. BrunNACKER 1964), sehr nahe. Aber erst jetzt ist es infolge der großräumig angelegten Arbeit von H. Körber (1962) sinnvoll geworden, ein geeignetes Profil des Maintales zu behandeln und zu diskuticren, nämlich das der Ziegeleigrube beim Bahnhof Marktheidenfeld, das bereits durch E. RutTE $(1956,1957)$ und H. KörBer (1962) erwähnt wird.

Man kann über diese Deckschichten und die darin entstandenen Paläoböden freilich nur das jeweilige Mindestalter der unterlagernden Bildungen festlegen - aber dieses bei richtiger Bodenansprache sehr genau. Dazu sind nicht einmal die Routineanalysen erforderlich, die paläopedologische Beiträge - meine eigenen nicht ausgenommen - gewöhnlich zu begleiten pflegen; denn das was heute durch die Geländebeobachtung hinsichtlich der bodengenetischen Probleme im Quartär nicht erkannt werden kann, wird zumeist auch nicht durch solche Laboratoriumsdaten erfaßt. Es soll damit jedoch keinesfalls zum Ausdruck gebracht werden, daß allgemein auf Analysen zu verzichten ist Wir müssen uns vielmehr endlich wieder daran gewöhnen, einerseits der Geländebeobachtung das nötige Vertrauen entgegenzubringen und andererseits den dabei auftauchenden speziellen Problemen durch der Fragestellung angepaßte zusätzliche Untersuchungen Rechnung zu tragen; denn nur auf diese Weise sind weitergehende Aussagemöglichkeiten zu erreichen. Im Falle der Interglazialböden Mitteleuropas dürfte es heute wenigstens bei einiger bodenkundlicher Schulung kein allzugroßes Problem mehr bedeuten, dieselben richtig zu deuten - handelt es sich doch hierbei ganz überwiegend um Böden vom Typus der sehr leicht erkennbaren Parabraunerde und deren Übergangsglieder zum Pseudogley.

Auf Gradabteilungsblatt Marktheidenfeld (Nr. 6123) liegt die Ziegeleigrube westlich des Mains etwa $300 \mathrm{~m}$ westlich vom Bahnhof Marktheidenfeld mit einer NW-SO verlaufenden $200 \mathrm{~m}$ langen oberen, im Löß angelegten Abbauwand; darunter sind außerdem die in früherer Zeit abgebauten Talsedimente erschlossen. Der Grubenaufschluß gliedert sich damit in drei Abteilungen, nämlich im Liegenden in die heute nicht mehr abgebauten Tone mit Torfzwischenlagen, darüber folgen Schotter und Sande mit bis $3 \mathrm{~m}$ und schließlich die heute im Abbau befindlichen Lößdeckschichten mit mehreren fossilen Böden. Damit erfaßt der Aufschluß (Abb. 1):

1. Einen Ausschnitt der alten Talverschüttung, die nach H. KörBER (1962, Abb. 2) bei Marktheidenfeld als sog. A-Terrasse (=Akkumulationsterrasse) insgesamt $45 \mathrm{~m}$ Mächtigkeit zwischen 150 und $195 \mathrm{~m}+\mathrm{NN}$ erreicht. Davon sind an der heutigen Wand rd. 5,5 $\mathrm{m}$ zwischen 164 und $170 \mathrm{~m}+\mathrm{NN}$ aufgeschlossen;

2. einen bis $3 \mathrm{~m}$ mächtigen Schotter, der im Niveau der nachfolgend darin wieder eingetieften E-Terrasse (=Erosionsterrasse) im Sinne von H. KöRBER (1962) mit Oberkante bei $173 \mathrm{~m}+\mathrm{NN}$ liegt;

3. auf dieser E-Terrasse lagern die bis $10 \mathrm{~m}$ mächtigen Deckschichten.

Nach Westen hin lehnt sich diese Verschüttung an den rd. $300 \mathrm{~m}+\mathrm{NN}$ ansteigenden Buntsandstein an. Östlich des Mains ziehen aus dem einige Kilometer entfernten Muschelkalkgebiet einige Seitentälchen zum Haupttal hin.

\section{Das Profil}

Das aufgenommene Profil zeigt den in Abb. 1 dargestellten und nachfolgend beschriebenen Aufbau: 
Tiefe in $\mathrm{m}$

\begin{tabular}{|c|c|c|}
\hline $0-0,20$ & $\begin{array}{l}\text { humushaltiger, braungrauer, bröckeliger, feinsan- } \\
\text { diger Lehm }\end{array}$ & \multirow{2}{*}{$\begin{array}{l}\text { rez. Boden }= \\
\text { Parabraunerde } \\
\text { aus Löß }\end{array}$} \\
\hline $0,20-0,30$ & $\begin{array}{l}\text { schwach humushaltiger, hellbraungrauer, bröcke- } \\
\text { liger, feinsandiger Lehm }\end{array}$ & \\
\hline $0,30-0,80$ & rötlichbrauner, brockiger, toniger Lehm & \multirow{5}{*}{$\begin{array}{l}\text { 1. foss. Boden = } \\
\text { schwach pseudo- } \\
\text { vergleyte Para- } \\
\text { braunerde aus } \\
\text { Löß }\end{array}$} \\
\hline $0,80-1,40$ & gelbgrauer, kalkreicher Löß & \\
\hline $1,40-1,80$ & $\begin{array}{l}\text { hellgelbgrauer, schluffiger Lehm, mit bis } 1 \mathrm{~cm} \\
\text { großen Eisenkonkretionen }\end{array}$ & \\
\hline $1,80-2,40$ & $\begin{array}{l}\text { braun und grau gefleckter, bröckeliger, toniger } \\
\text { Lehm, rostfleckig }\end{array}$ & \\
\hline $2,40-3,10$ & $\begin{array}{l}\text { rötlichbrauner, brockiger, toniger Lehm, graue } \\
\text { Verfahlungen längs einzelner Klüfte }\end{array}$ & \\
\hline $3,10-3,50$ & hellbrauner, kalkhaltiger Löß & \\
\hline $3,5,0-4,00$ & $\begin{array}{l}\text { hellgelbgrauer, schluffiger Lehm, unten mit steck- } \\
\text { nadelkopfgroßen Eisenkonkretionen }\end{array}$ & \multirow{4}{*}{$\begin{array}{l}\text { 2. foss. Boden = } \\
\text { pseudovergleyte } \\
\text { Parabraunerde } \\
\text { aus Löß (im BC- } \\
\text { Horizont ist } \\
\text { außerdem der } \\
\text { unterlagernde } \\
\text { A3g-Horizont } \\
\text { etwas erfaßt) }\end{array}$} \\
\hline $4,00-4,40$ & $\begin{array}{l}\text { braun und grau gefleckter, bröckeliger, toniger } \\
\text { Lehm, rostfleckig }\end{array}$ & \\
\hline $4,40-5,50$ & $\begin{array}{l}\text { rötlichbrauner, brockiger, toniger Lehm, schwach } \\
\text { graufleckig }\end{array}$ & \\
\hline $5,50-5,80$ & $\begin{array}{l}\text { gelbgrauer, schluffiger Lehm, mit } 1 \mathrm{~cm} \text { dicken rost- } \\
\text { braunen Bändern }\end{array}$ & \\
\hline
\end{tabular}

A3g 5,80- 6,10 gelbgrauer, bröckeliger, schluffiger Lehm, mit stecknadelkopfgroßen Eisenkonkretionen

$\mathrm{Bg}$ 6,10-6,30 rötlichbrauner, brockiger, toniger Lehm, grau- und rostfleckig

gB 6,30-7,10 rötlichbrauner, brockiger, toniger Lehm, schwach grau- und rostfleckig

gBC 7,10-9,00 graugelber, feinsandiger Schluff, mit zentimeterdicken rostbraunen Bändern, graufleckig

C 9,00-9,50 hellbrauner, kalkhaltiger Schluff (Löß?), oben mit gänseeigroßen Lößkindeln (Horizont keilt seitlich aus)

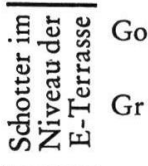

$9,50-10,5,0$

$10,50-12,50$

Erosionsdiskordanz

$12,50-13,5,0$

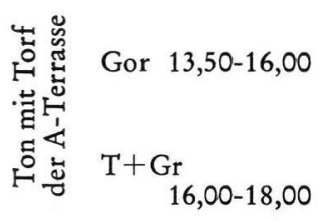

sandiger Schotter, oben einzelne kantige Schollen, mit dezimeterdicken rostbraunen Bändern

hellgraubrauner sandiger Schotter, nach oben in kiesigen Sand übergehend
3. foss. Boden $=$ pseudovergleyte Parabraunerde aus Löß
4. foss. Boden $=$ Gley aus sandigem Schotter

hellgrauer Ton, mit bis $5 \mathrm{~cm}$ dicken rostbraunen Schlufflinsen (umgelagertes Material des 5. fossilen Bodens) $\left.\begin{array}{l}\text { hellgrauer, schluffiger Ton, mit einzelnen Rost- } \\ \text { flecken }\end{array}\right\}$

5. foss. Boden $=$ Gor-Hor. eines Gleyes

dunkelgrauer, schwach humushaltiger Ton, besonders oben mit bis $40 \mathrm{~cm}$ dicken torfigen Lagen (z. B. Schwemmtorf mit Hölzern)

6. foss. Boden $=$ $\mathrm{Gr}$-Hor. eines

Gleyes und Niedermoortorf 
a) Die Siedimente der Talverschüttung

Die in der Grube aufgeschlossenen Tone und erst recht die torfigen Lagen stellen lokal begrenzte Einschaltungen innerhalb der Gesamtverschüttung (=A-Terrasse) dar. Etwa ab Haßfurt läßt sich diese Verschüttung talabwärts durchverfolgen. Im Liegenden wird sie nach H. KörBer (1962) durch beim Bahnhof Marktheidenfeld aufgeschlossene Schotter eingeleitet. Die Hauptmasse dieser Sedimente besteht jedoch allgemein aus mehr oder weniger kiesigen Sanden, in welche das Tonlager zwischengeschaltet ist. Dieses Tonlager wurde von H. Körber (1962) zwischen Marktheidenfeld und Miltenberg festgestellt, wo die Gesamtmächtigkeit der Verschüttung schließlich bis $\mathrm{rd} .65 \mathrm{~m}$ erreicht.

Im Bereich der dem Ton eingelagerten Torflinsen bei Marktheidenfeld haben die von S. HEYDENREICH durchgeführten botanischen Untersuchungen ein altquartäres Alter mit interglazialem Klima erbracht.')

\section{b) Die E-Terrasse}

Nach der Verschüttung kam es infolge Tieferlegung des Talbodens zur Herausarbeitung der E-Terrasse, die nach $H$. KönBER (1962) etwa ab Karlstadt mainabwärts nachzuweisen ist. Im Niveau dieser Fläche befinden sich die um $170 \mathrm{~m}+\mathrm{NN}$ lagernden Schotter der Marktheidenfelder Grube. Insgesamt handelt es sich dabei in diesem Aufschluß um wohlgerundete Gerölle aus Buntsandstein mit einer Durchschnittsgröße von $2 \mathrm{~cm}$, mit sandigem Zwischenmittel. Vom Liegenden nach dem Hangenden nimmt der Sandanteil zuerst zu und geht dann schließlich wieder in entsprechende Schotter über. Lydite und Quarze sind vorhanden. Der Muschelkalk ist hingegen nur durch verkieseltes Material vertreten. Die Kalke fehlen primär, obwohl Muschelkalkmaterial aus nächster Nähe hätte zugeliefert werden können. Damit scheidet auch für diese Schüttung eine ausgesprochen kaltklimatische Stellung aus. Lediglich bis $50 \mathrm{~cm}$ große, kantige Buntsandsteinschollen nächst der Hangendgrenze dieses Schotters könnten ein Hinweis auf ein zwischendurch wieder etwas geändertes Klima sein.

Reste des Go-Horizontes eines Gleyes deuten nachfolgend auf eine Bodenbildung mit Grundwasserstand nächst der Oberfläche.

Am NW-Ende des Aufschlusses liegen im Niveau dieses Schotterlagers jedoch andersartige Sedimente, nämlich ein sandiger, nur mäßig gerundeter Grobschotter aus Buntsandsteinmaterial mit $10-20 \mathrm{~cm}$ Gerölldurchmesser und kantengerundeten Blöcken von bis ${ }^{1 / 4} \mathrm{~m}^{3}$ Größe. Nach dem Hangenden geht an dieser Stelle das Zwischenmittel in roten, umgelagerten Buntsandsteinton über. Obwohl dieser Bereich nicht besonders gut aufgeschlossen ist, zeigt sich ein gegen das Maintal gerichtetes Oberflächengefälle an, womit sich diese Einschaltung als die Schüttung eines Seitentälchens erklären läßt, das aus nordwestlicher Richtung gekommen ist und das heute etwa $1 / 2 \mathrm{~km}$ weit in das Buntsandsteingebiet hinein verfolgt werden kann, das aber möglicherweise ursprünglich über einen anderen Verlauf und ein größeres Einzugsgebiet (des heutigen Glasbaches) verfügte. Dem Habitus nach handelt es sich um einen Wildbachschutt, der nach oben in eine Fließerde bzw. in eine Mure übergeht. Eine Verzahnung mit dem Schotter der E-Terrasse konnte nicht beobachtet werden. Es wäre höchstens denkbar, daß die an dessen Obergrenze liegenden Buntsandsteinschollen, die aber wiederum deutlich scharfkantiger ausgebildet sind als die des Wildbachschuttes, dem Schutt des Seitentälchens entsprechen. Dann aber müßte auch eine Überprägung des roten Tones im Rahmen der genannten Vergleyung stattgefunden haben. Davon ist aber nichts zu erkennen. Diese Einschaltung wird deshalb folgendermaßen ge-

1) Es handelt sich hierbei um eine bisher nicht veröffentlichte Staatsexamensarbeit. Nach freundlicher mündlicher Mitteilung von Frau LaUfer-Heydenreich, München, wurde die gefundene Flora in das Günz/Mindel-Interglazial eingestuft. 
deutet: Nach Ablagerung des Schotters im Niveau der E-Terrasse und Gley-Bildung kam es im Haupttal wieder zu einer gewissen Eintiefung des Talbodens. Diese hatte ein Einschneiden des Seitentälchens und damit auch eine in den Schotter der E-Terrasse eingetiefte Erosionsrinne zur Folge. Diese Rinne wurde anschließend mit Bachschutt des Seitentälchens gefüllt. Belanglos ist dabei, ob dessen hangende Partien eine Mure oder einen Solifluktionsschutt darstellen. Sie leiten in jedem Falle in die Deckschichten des Profils über.

c) Die Deckschichten

Auf die Deckschichten im einzelnen einzugehen, erübrigt sich. Es handelt sich um vier Lösse, von denen der unterste allerdings etwas problematischer Natur ist, mit drei zwischengeschalteten Interglazialböden vom Typ der Parabraunerde und damit um eine dem Regensburger Profil vergleichbare Abfolge. Lediglich die Lösse zeigen entsprechend ihrer Lage im mainfränkischen Trockengebiet eine weniger feuchte Fazies an und dementsprechend sind auch diese Parabraunerden mit sehr großer Entwicklungstiefe etwas weniger stark pseudovergleyt. Konsequenterweise ergibt sich damit für die unterlagernde alte Mainverschüttung ebenfalls eine in die Zeit vor der viertletzten Eiszeit (und damit vor der Günzeiszeit) gehörige Altersstellung.

\section{Beziehungen der Talverschüttung zu den Terrassen des Mains und der Donau}

Oben wurde darauf verwiesen, daß die Deckschichten normalerweise nur das Mindestalter der unterlagernden Terrassenkörper zu liefern vermögen. Bei jüngeren Terrassenflächen mit zahlreichen Aufschlüssen im Deckschichtenbereich ist dennoch zumeist mit genügend großer Genauigkeit eine Aussage über das Alter des Liegenden möglich. Für Marktheidenfeld gilt dies als Einzelaufschluß jedoch ebenso wenig wie für Regensburg, wenngleich an beiden Stellen keine Erosionsdiskordanz innerhalb der Deckschichtenfolge an den sehr langen Aufschlußwänden zu erkennen ist. Es ist deshalb nötig, so weit wie möglich noch andere Alterskriterien heranzuziehen.

\section{a) Die jüngeren Terrassen}

Bei Regensburg fehlt vorläufig ein Hinweis auf die günzeiszeitliche Terrasse (vgl. K. BRunNaCKen 1964); hier vermittelt also die Deckschichtenfolge eine genauere Aussage als die Terrassenfolge. Im näheren Umkreis von Marktheidenfeld ist nach H. KörbER (1962) nur die sog. Obere Mittelterrasse und die Niederterrasse ausgebildet. Insgesamt aber hat $H$. KöRBer im Maintal vier Terrassen festgestellt, die jünger sind als die Talverschüttung. Sie lassen sich $\mathrm{m}$. E. zumindest theoretisch folgendermaßen einstufen, wobei allerdings die an die Terrassen des Rheintales angelehnte Bezeichnungsweise von $\mathrm{H}$. KöRBER keinesfalls eine entsprechende Übereinstimmung wiederzugeben braucht - bestehen $\mathrm{z}$. T. auch abweichende Auffassungen hinsichtlich der Altersstellung (vgl. A. KEssLen 1962):

$$
\begin{array}{ll}
\text { Obere Mittelterrasse } & =\text { Günz } \\
\text { Mittlere Mittelterrasse } & =\text { Mindel } \\
\text { Untere Mittelterrasse } & =\text { Riß } \\
\text { Niederterrasse } & =\text { Würm. }
\end{array}
$$

\section{b) Die älteren 'Terrassen}

Im Regensburger Raum deutet sich durch Schotterschleier die Existenz von wenigstens zwei älteren Quartärterrassen an; dazu kommt ein bereits in das Pliozän gestellter Schotter. Im Maintal läßt sich nach H. KörBER (1962) eine altersmäßig der Verschüttung vorangehende obere, mittlere und untere Hauptterrasse ausscheiden. 
In der Zeit unmittelbar vor der Talverschüttung müßte nach KöRBER im Untermaingebiet eine Eintiefung des Tales um 70-80 m unter das Niveau der Unteren Hauptterrasse erfolgt sein. Anschließend ist es dann im Eintiefungsgebiet wieder zu der bis $65 \mathrm{~m}$ mächtigen Talverschüttung gekommen, wobei bemerkenswert ist, daß letztere etwa ab Obernburg das im Maintal liegende Oberpliozän überdeckt. Nachdem im Untermaingebiet die Hauptterrassen nicht wie im Mittel- und Obermaingebiet durch Schotter, sondern nur durch Flächenniveaus - also doch etwas problematisch - belegt sind, wäre es denkbar, daß die schotterführenden echten Hauptterrassenniveaus mainabwärts unter die zunehmend mächtiger werdende Verschüttung hinabtauchen. Es wäre damit im Untermaingebiet der Verschüttung keine Zeit vorausgegangen, die durch einen besonders großen Eintiefungsbetrag gekennzeichnet war.

\section{Vergleich mit der Talverschüttung bei Regensburg}

Sowohl in Regensburg (vgl. K. BRUnNACKer 1964) als auch in Marktheidenfeld kommt es im Anschluß an eine Erosionsphase zur durch Schotter eingeleiteten Talverschüttung; darüber folgt Sand bzw. sandig-tonige Sedimente. Dem „Auenboden“ in Regensburg entspricht dann die als „Auesediment" aufgefaßte, z. T. torfige Tonlage im Maintal. Darüber kommt in beiden Fällen eine zweite Ablagerungsphase von sandig-tonigen Sedimenten bzw. Sand.

Die weitere Entwicklung ist dadurch gekennzeichnet, daß in Regensburg jetzt ein Komplex erfaßt ist, der in Marktheidenfeld an und über der Oberfläche der hier nicht aufgeschlossenen A-Terrasse zu suchen wäre. In Marktheidenfeld ist hingegen eine auf der E-Terrasse lagernde Abfolge erfaßt. Es folgt deshalb in Regensburg ein „extremer Pseudogley", in Marktheidenfeld hingegen „Erosion“ und der vermutlich dazugehörige, weil erodierend wirkende untere Teil des im Erosionsniveau liegenden Schotters.

In Regensburg kommen darüber „Abschwemm-Massen“; in Marktheidenfeld entspricht diesen der „obere Teil des Schotters im Niveau der E-Terrasse“, der kantige Buntsandsteinschollen führt. Man könnte dieser Phase auch den Bachschutt des Seitentälchens in Marktheidenfeld zuordnen; im Prinzip würde sich damit jedoch nichts ändern.

In Regensburg kam es anschließend zur Bildung eines „Pseudogleyes“ und in Marktheidenfeld eines "Gleyes“.

Infolge weiterer Eintiefung des Haupttales entstand dann in Regensburg eine große Erosionsdiskordanz, und aus dem gleichen Grund hat sich in Marktheidenfeld die Sohle des kleinen Seitentälchens eingeschnitten.

In Regensburg folgt darüber die die Günzeiszeit einleitende Basisfließerde, in Marktheidenfeld hingegen der Bachschutt und darïber die Fließerde mit Material des Buntsandsteins.

Im großen und ganzen liegen also bei Regensburg und Marktheidenfeld entsprechende Beziehungen zwischen Verschïttung und Terrassenfolge vor. Darin kann ein weiterer Hinweis auf die gleichaltrige Stellung derselben im Entwässerungssystem des Mains wie der Donau gesehen werden. Die bei Regensburg zur Deutung der Verschüttung u. a. heranziehbare Lokaltektonik verliert infolgedessen erheblich an Wert zugunsten großräumiger Hebungen der Mittelgebirgsareale.

Damit besteht sehr weitgehende Vergleichbarkeit des Ablaufes der Talverschüttung in völlig verschiedenen Talsystemen, und es deutet sich die in Tab. 1 gegebene Gliederung im Prinzip auch dann an, wenn unterstellt wird, daß bei zunehmender Kenntnis über diese alte Talverschüttung manche Einzelheiten einer Ergänzung bzw. Berichtigung bedürfen. 
Tabelle 1

Gliederung und klimatische Bewertung der Talverschüttungsfolge in Marktheidenfeld und Regensburg

Abfolge in Regensburg und Marktheidenfeld klimatische Deutung

9. Bachschutt und Fließerde (=Beginn der Günzeiszeit)

8. Erosion im Haupttal

Gley bzw. Pseudogley

7. Schwemmschicht bzw. Wildbachschutt

6. extremer Pseudogley bzw. Erosion und Schotterlage

5. tonige und sandige Flußsedimente

4. Aueboden bzw. Auesediment

3. tonige und sandige Flußsedimente

2. Erosion und Basisschotter

1. Untere Hauptterrasse (=letzte Donaukaltzeit?)

kalt - feucht
kühl - relativ feucht
kühl - relativ trocken
warm - feucht
relativ kühl - relativ trocken
warm - feucht
relativ kühl - relativ trocken
warm - feucht
kalt?

\section{Die klimatische Bewertung der Talverschüttung}

Vermutlich handelt es sich beim liegenden Schotter (Tab. 1, Nr. 2) um Reste des Geröllmaterials, das zur Herausarbeitung des Talzuges in der vorangegangenen Erosionsphase nötig war. Damit ist der mit der ersten Erosionsphase (Nr. 2) beginnende Abschnitt bis einschließlich der Herausbildung der E-Terrasse (Nr. 6) als eine sehr lange Warmzeit zu deuten, jedoch gegliedert durch Zeiten mit etwas abweichendem Klima.

Wenn man nämlich von einer in den Tälern ungefähr gleichbleibenden Tendenz zur Sedimentanlieferung ohne entscheidende Beteiligung von Periglazialschutt infolge geschlossener Vegetationsdecke in der Gesamtzeit (Nr. 2 bis 8) ausgeht, dann waren feuchtere Abschnitte normalerweise durch Neigung zur Erosion und trockenere (auch etwas kühlere?) Abschnitte durch Akkumulation gekennzeichnet. Am Anfang steht dann eine feuchtere Zeit (Nr. 2), die von einer trockeneren abgelöst wurde (Nr. 3). Dieser Wechsel hat sich mehrmals wiederholt, jedoch modifiziert durch vor allem in Abschnitt Nr. 4 und 5 wirksam werdenden Aufstau infolge verstärkter Hebung der Mittelgebirgsbereiche, die vom Main bzw. der Donau durchsägt werden mußten. Erst über dem Basisschotter im Bereich der Marktheidenfelder E-Terrasse bzw. in der Regensburger Schwemmschicht über dem extremen Pseudogley (Nr. 7) finden sich Hinweise auf eine zunehmende Klimaverschlechterung. Die hangenden Fließerden und Bachablagerungen bilden dann schließlich den Übergang zur Günzeiszeit.

\section{Beziehung der Talverschüttung zu den altquartären Säugetierfaunen Südwestdeutschlands}

Bei Randersacker wurde in dieser Talverschüttung eine von E. RutTE (1958) bearbeitete Säugetierfauna gefunden, die eher der Jüngeren Steppenzeit (=Mittlere Stufe der Mosbacher Sande) als der Mauerer Waldzeit entspricht. Der Sedimentationsbeschreibung von E. Rutre (vgl. insbesondere 1959) nach handelt es sich bei Randersacker um Bildungen, die vermutlich unter relativ trockenen Bedingungen dem jüngeren Geschehnisablauf von Marktheidenfeld (z. B. Nr. 5 und 7, vielleicht sogar noch 9) entsprechen. Die als älter anerkannte Mauerer Waldzeit im Sinne von K. D. ADAM (1953) könnte dann der Auephase mit Ton- und Torfablagerung bei Marktheidenfeld gleichgesetzt (Nr. 4) oder gegebenenfalls noch etwas jünger eingestuft werden (in Nr. 6).

An dieser Stelle sei auch an den von I. VALETON (1956) etwas oberhalb Würzburg an der Sohle des Maintals gefundenen fossilen Boden aus Muschelkalk erinnert. Sicher liegt dieser fossile Boden in $170 \mathrm{~m}+\mathrm{NN}$ in einem tieferen Niveau als die in der Nähe gefundenen Großsäugerreste (in 
185-190 m + NN). Nachdem in diesem Gebiet auch H. KöRBER (1962) die Basis der alten Verschüttung zwischen 170 und $180 \mathrm{~m}+\mathrm{NN}$ angibt, spricht manches dafür, daß der von J. VALETON (1956) beschriebene, in seiner typologischen Deutung jedoch problematische Boden in die Anfangszeit der Talverschüttung gehört, in der sich die Akkumulation im Mittelmaingebiet noch nicht ausgewirkt hat.

Von E. Rutte (1957) wird die Fauna von Randersacker gemäß K. D. AdAM (1953) in den Beginn der Mindeleiszeit eingestuft. Es müßte aber genauer lauten: In den Beginn der Elsterzeit; denn üblicherweise werden die südwestdeutschen Säugetierfaunen auf die norddeutschen und nicht auf die alpine Gliederung des Quartärs bezogen. Infolgedessen ist es derzeit auch noch sehr schwierig, die stratigraphische und paläoklimatische Auswertung der Faunenfolge der altquartären Großsäuger damit in Übereinstimmung zu bringen. Eher lassen sich hingegen Parallelen zwischen der stärker gegliederten altquartären Kleinsäugerabfolge (vgl. Fl. Heller 1958) und der in diesem Beitrag geschilderten SedimentBoden-Folge, die sicherlich noch nicht unmittelbar an das Pliozän anschließt, erkennen. Das geologische und paläopedologische Ziel muß schließlich die genauere Festlegung der Stellung dieses prägünzeiszeitlichen „Interglazials mit dem Riesenboden“, wie es ursprünglich genannt wurde (vgl. K. Brunnacker 1962), zu den Donaueiszeiten und zum Villafranchium sein, das m. E. im Bereich der Hauptterrassen zu suchen ist.

\section{$\mathrm{Schrift} \mathrm{tum}$}

Adam, K. D.: Die Bedeutung der altpleistozänen Säugetier-Faunen für die Gliederung des Eiszeitalters. - Geologica Bavarica, Nr. 19, S. 357-363, München 1953.

Brunnacker, K.: Pleistozäne Böden im nördlichen Oberschwaben. In Graul, H.: Eine Revision der pleistozänen Stratigraphie des schwäbischen Alpenvorlandes, S. 255-259, Petermanns geograph. Mitt., 1962, S. 253-271, Gotha 1962. - - Böden des älteren Pleistozäns bei Regensburg. - Geologica Bavarica, Nr. 53, S. 148-160, München 1964.

Heller, Fl.: Eine neue altquartäre Wirbeltierfauna von Erpfingen (Schwäbische Alb). - Neues Jb. Geol. u. Paläont., Abh. 107, S. 1-102, Stuttgart 1958.

Hirsch, L.: Diluviale Tektonik im Maintal bei Würzburg. - Oberrhein. geol. Abh., 11, S. 44-63, Karlsruhe 1940.

Kessler, A.: Studien zur jüngeren Talgeschichte am Main und an der Mümling und über jüngere Formenentwicklung im hinteren Buntsandstein-Odenwald. - Forsch. Dt. Landeskde., 133, Bad Godesberg 1962.

Körber, H.: Die Entwicklung des Maintals. - Würzburger geograph. Arb., 10, Würzburg 1962.

RutTE, E.: Zur Geologie in Unterfranken. - Hierzu drei Exkursionen. - Z. deutsch. geol. Ges., 108, S. 73-87, Hannover 1956. - - Einführung in die Geologie von Unterfranken. Würzburg 1957. - - Die Fundstelle altpleistozäner Säugetiere von Randersacker bei Würzburg. - Geol. Jb., 73, S. 737-754, Hannover 1958. - - Neue Beobachtungen in der Fundstelle altpleistozäner Säugetiere von Randersacker bei Würzburg. - Fränkische Natur u. Landschaft, H. 2, S. 30-35, Würzburg 1959.

Valeton, I.: Fossile Bodenbildungen an der Sohle des Maintals. - Geologica Bavarica, Nr. 25, S. 44-50, München 1956.

Wurm, A.: Beiträge zur Flußgeschichte des Mains und zur diluvialen Tektonik des Maingebietes. Geologica Bavarica, Nr. 25, S. 1-21, München 1956.

Manuskr. eingeg. 5. 12. 1963.

Anschrift des Verf.: Prof. Dr. K. Brunnacker, Geologisches Institut der Universität, 5 Köln, Zülpicher Straße 49. 